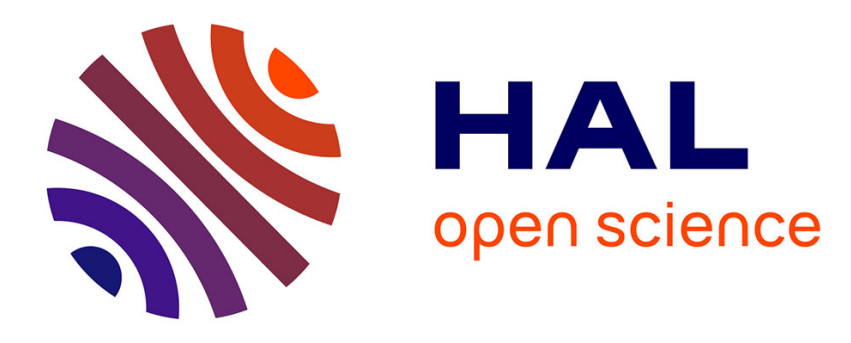

\title{
Riccati observers for position and velocity bias estimation from direction measurements
}

\author{
Tarek Hamel, Claude Samson
}

\section{To cite this version:}

Tarek Hamel, Claude Samson. Riccati observers for position and velocity bias estimation from direction measurements. CDC 2016 - Conference on Decision and Control, Dec 2016, Las Vegas, United States. hal-01426900

\author{
HAL Id: hal-01426900 \\ https://hal.science/hal-01426900
}

Submitted on 5 Jan 2017

HAL is a multi-disciplinary open access archive for the deposit and dissemination of scientific research documents, whether they are published or not. The documents may come from teaching and research institutions in France or abroad, or from public or private research centers.
L'archive ouverte pluridisciplinaire HAL, est destinée au dépôt et à la diffusion de documents scientifiques de niveau recherche, publiés ou non, émanant des établissements d'enseignement et de recherche français ou étrangers, des laboratoires publics ou privés. 


\title{
Riccati observers for position and velocity bias estimation from direction measurements
}

\author{
Tarek Hamel and Claude Samson
}

\begin{abstract}
This paper addresses the problem of estimating the position of a body moving in $n(\geq 2)$-dimensional Euclidean space using body velocity measurements and the measurements of direction(s) between the body and one (or several) source point(s) whose location(s) is (are) known. The proposed solutions exploit the Continuous Riccati Equation (CRE) to calculate observer gains yielding global uniform exponential stability of zero estimation errors, even when the measured body velocity is biased by an unknown constant perturbation. These results are obtained under persistent excitation (p.e.) conditions depending on the number of source points and body motion that ensure both uniform observability and good conditioning of the CRE solutions. With respect to previous contributions on the subject the proposed framework encompasses the static case, when the body is motionless and at least two source points are needed to recover its position, and the non-static case, when body motion and a single source are sufficient. Simple and explicit observability conditions under which uniform exponential stability is achieved are also worked out for each case. Simulation results illustrate the performance of the proposed observers.
\end{abstract}

\section{INTRODUCTION}

The general problem of estimating the position, or the complete pose (position and orientation), of a body relatively to a certain spatial frame is central for a multitude of applications. This is common knowledge. Among all sensing modalities that can be used to acquire the necessary information, source points direction (or bearing) measurements has early motivated many studies, in particular for pose estimation when body and source points are motionless in the frame of interest, a problem referred to as the Perspective$n$-Point (PnP) problem in the dedicated literature [1]. The present paper focuses on the sole estimation of the body position. This corresponds to applications for which the body's attitude is either of lesser importance or is estimated by using other sensing modalities. In this case, iterative and recursive methods using ongoing measurements information, by opposition to non-iterative ones based on the algebraic resolution of a finite set of constraint equations, are all the more interesting that their domain of convergence can be global. The reason is that, without the compact group of rotations being involved, this simplified problem is amenable to exact linearisation and can be associated with globally convex cost functions, as shown further in the paper. Another advantage of iterative methods is that they are naturally suited to handle the non-static case, i.e. when either the body

T. Hamel is with I3S UNS-CNRS, Nice-Sophia Antipolis, France, thamelei3s.unice.fr.

C. Samson is with INRIA and I3S UNS-CNRS, Sophia Antipolis, France, claude.samsoneinria.fr, csamsonei3s.unice.fr. or the point source(s) move(s), by using on-line the extra data and information resulting from motion. In particular, the observation of a single point source may be sufficient in this case, provided that the body motion regularly grants a sufficient amount of "observability". This possibility has been studied recently in [2] where the problem is linearised by considering an augmented state vector. Another solution, not resorting on state augmentation, is proposed in [3]. The present paper offers a generalization of previous studies on this subject that encompasses the static and non-static cases with an arbitrary number of source points.

For five decades, Kalman filters [4] for linear systems, and their extensions to non-linear systems known as Extended Kalman Filters (EKF) [5], have consistently grown in popularity near engineers with various backgrounds (signal processing, artificial vision, robotics,...) to address a multitude of iterative state estimation problems involving additive "noise" upon the state and/or the measurements. The optimality of these filters in a stochastic framework under specific noise conditions and assumptions, and their direct applicability to Linear Time-Varying (LTV) systems, have undoubtedly contributed to this popularity. It is however important to keep in mind, or to recall, that the stability and robustness properties associated with them, i.e. features that supersede conditional stochastic optimality in practice, are not related to stochastic issues. They result from properties of the associated deterministic continuous-time (or discrete-time, depending on the chosen computational framework) Riccati equation that underlies a (locally) convex estimation error index (or Lyapunov function) and a way of forming recursive estimation algorithms that uniformly decrease this index exponentially (under adequate observability conditions). With this perspective, Kalman filters belong to the (slightly) larger set of Riccati observers that we intentionally derive here in a deterministic framework, knowing that a complementary stochastic interpretation may be useful to subsequently tune the Riccati equation parameters and observer gains. We also believe that, by contrast with standard Kalman filter derivations, this approach allows one to better comprehend how the system observability properties are related to the good conditioning of the Riccati equation solutions and to the observer's performance (the rate of convergence to zero of the estimation errors, in particular) via a Lyapunov analysis.

The paper is organized along five sections. Following the present introduction, Section II specifies some of the notation used throughout the paper, recalls the equations of a generic Riccati observer associated with a LTV system, as well as a few central properties of the corresponding CRE, and states 
complementary technical results tailored for the design and analysis of the observers here proposed. Position estimation from direction measurements in $n(\geq 2)$-dimensional Euclidean space is specifically treated in Sections III. A single source point is first considered, with stability and convergence of the observer relying on persistent excitation properties associated with the body motion. Solutions are then generalized to the case of multiple source points, with the augmentation of the number of these points reflecting in the gradual weakening of the body motion conditions needed to ensure uniform exponential stability. While measuring the body velocity is central to estimate the position, we show how to modify the observers via state augmentation when velocity measurement are biased by a constant vector. We also show that uniform exponential stability is preserved under the same observability conditions. Illustrative simulation results are presented in Section IV, followed by a short section V of concluding remarks.

\section{RICCATI OBSERVERS}

Throughout the paper the following notation is used:

- $A(t), B(t), C(t)$ are finite-dimensional matrix-valued functions depending on time. They are continuous and bounded.

- The abbreviation p.s.d. (resp. p.d.) is used to denote semipositive (resp. positive) square matrices that are also symmetric. Square null matrices are p.s.d. matrices and identity matrices, denoted as $I_{d}$ independently of their dimensions, are p.d. matrices.

- $Q(t)$ and $V(t)$ are p.s.d. finite-dimensional matrixvalued functions of time. They are also continuous and bounded. When no specific indication is provided in the text these matrix-valued functions are chosen strictly positive and greater than $\epsilon I_{d}$ with $\epsilon>0$.

- The infimum (resp. supremum) over time of the smallest (resp. largest) eigenvalue of a p.s.d matrix-valued function $P(t)$ is denoted as $p_{m}$ (resp. $p_{M}$ ). For the matrixvalued function $V(t)$ these infimum and supremum values are accordingly denoted as $v_{m}$ and $v_{M}$.

\section{A. Riccati observers}

Consider a generic linear time-varying (LTV) system

$$
\left\{\begin{aligned}
\dot{x} & =A(t) x+B(t) u \\
y & =C(t) x
\end{aligned}\right.
$$

with $x \in \mathbb{R}^{n}$ the system state vector, $u \in \mathbb{R}^{s}$ the system input vector, and $y \in \mathbb{R}^{m}$ the system output vector. We here call Riccati observer any observer of System (1) of the form

$$
\dot{\hat{x}}=A(t) \hat{x}+B(t) u+K(t)(y-C(t) \hat{x}) ; \hat{x}(0) \in \mathbb{R}^{n}
$$

with the observer gain given by

$$
K(t)=k(t) P(t) C^{\top}(t) Q(t) ; k(t) \geq 0.5
$$

where $P(t)$ is the solution to the so-called Continuous Riccati Equation (CRE)

$$
\dot{P}=A(t) P+P A^{\top}(t)-P C^{\top}(t) Q(t) C(t) P+V(t)
$$

with $P(0)$ any p.d. matrix and $Q(t), V(t)$ p.s.d. matrices that have to be specified. Note that the optimal Kalman gain in the stochastic setting where the matrices $V(t)$ and $Q^{-1}(t)$ are p.d. matrices and interpreted as covariance matrices of additive noise on the system state and output is obtained by taking $k(t)=1$.

Let us now quickly recall how the stability and convergence properties of a Riccati observer is directly related to the properties of the solution $P(t)$ to the CRE. Define the estimation error $\tilde{x}:=x-\hat{x}$, from (1) and (2) one obtains the error equation

$$
\dot{\tilde{x}}=(A(t)-K(t) C(t)) \tilde{x}
$$

Assume (for the time being) that $P(t)$, which is a symmetric matrix by construction, is well defined on $\mathbb{R}^{+}$and is p.d., so that its inverse is also well defined and p.d., and consider the candidate Lyapunov function $\mathcal{V}(t)=\tilde{x}{ }^{\top}(t) P^{-1}(t) \tilde{x}(t)$. Then, using the fact that $\dot{P}^{-1}=-P^{-1} \dot{P} P^{-1}$, the timederivative of $P^{-1}$ satisfies the equation

$\dot{P}^{-1}=-P^{-1} A(t)-A^{\top}(t) P^{-1}+C^{\top}(t) Q(t) C(t)-P^{-1} V(t) P^{-1}$

and, using (3) and (5), one easily verifies that the timederivative of $\mathcal{V}(t)$ is given by

$$
\begin{aligned}
\dot{\mathcal{V}} & =-\tilde{x}^{\top}\left((2 k(t)-1) C^{\top}(t) Q(t) C(t)+P^{-1} V(t) P^{-1}\right) \tilde{x} \\
& \leq-\tilde{x}^{\top} P^{-1} V(t) P^{-1} \tilde{x} \\
& \leq-\frac{p_{m}^{2}}{p_{M}} v_{m} \mathcal{V} \quad(\leq 0)
\end{aligned}
$$

so that $\mathcal{V}(t) \leq \mathcal{V}(0) \exp \left(-\frac{p_{m}^{2}}{p m} v_{m} t\right)$. To conclude that $\tilde{x}=0$ is globally uniformly exponentially stable it thus suffices to choose $V(t)>v_{m} I_{d}$ with $v_{m}>0$ and to show that $P(t)$ i) is always well-defined, ii) that it is p.d., and -most importantlyiii) that it is well conditioned in the sense that $p_{m}$ is strictly positive and $p_{M}$ is finite so that the ratio $\frac{P_{M}}{p_{m}^{2}}$ is bounded. The central issue of boundedness and good conditioning of $P(t)$ brings us to recall classical, and also point out less known, results concerning the CRE.

\section{B. Properties of the Continuous Riccati Equation}

The first results concerns the existence of the solutions to the CRE for $t \in[0,+\infty)$.

Lemma 2.1: If $P(0)$ is p.d. and $Q(t)$ and $V(t)$ are p.s.d, then $P(t)$ is p.d. and well defined on $[0,+\infty)$.

The proof is given in the appendix A.

Now, to ensure boundedness and good-conditioning of the solution $P(t)$ to the CRE one has to impose other conditions upon the terms entering the equation. Sufficient conditions are pointed out in the next lemma.

Lemma 2.2: Define:

$$
W_{V}(t, t+\delta):=\frac{1}{\delta} \int_{t}^{t+\delta} \Phi(t, s) V(s) \Phi^{\top}(t, s) d s
$$

and

$W_{Q}(t, t+\delta):=\frac{1}{\delta} \int_{t}^{t+\delta} \Phi^{\top}(s, t) C^{\top}(s) Q(s) C(s) \Phi(s, t) d s$

with $\Phi(t, s)$ denoting the transition matrix associated with $A(t)$, i.e. such that $\frac{d}{d t} \Phi(t, s)=A(t) \Phi(t, s)$ with $\Phi(t, t)=$ 
$I_{d}$. If there exist (strictly) positive numbers $\delta, \mu_{v}$, and $\mu_{q}$ such that $W_{V}(t, t+\delta) \geq \mu_{v} I_{d}$ and $W_{Q}(t, t+\delta) \geq \mu_{q} I_{d}$, $\forall t$, then the solution $P(t)$ to the CRE (4) is bounded and well-conditioned in the sense that $0<p_{m} \leq p_{M}<\infty$.

A proof of this result is, for instance, given in [6] where lower and upper bounds of $P(t)$ are also derived. In this reference the matrix $Q(t)$ is in fact assumed p.d. because the inverse of $Q$ is (for technical convenience) used in the proof. However, it is simple to verify that the proposed bounds for $P(t)$ do not depend on the smallest eigenvalue of $Q(t)$, so that these bounds are also valid when $Q(t)$ is only p.s.d.

From now on, and by analogy with the classical observability Grammian

$$
W(t, t+\delta):=\frac{1}{\delta} \int_{t}^{t+\delta} \Phi^{\top}(s, t) C^{\top}(s) C(s) \Phi(s, t) d s
$$

$W_{Q}$ is called Riccati observability Grammian. It coincides with $W$ when $Q(t)=I_{d}$. Note that if $Q(t) \geq \epsilon I_{d}>0$ and the observability Grammian $W$ is positive then the Riccati observability Grammian $W_{Q}$ is also positive. This is just a consequence of that $W_{Q}(t, t+\delta) \geq \lambda_{\min }(Q(t)) W(t, t+\delta)$. Also, when the output matrix $C(t)$ varies with the the state trajectory $x(t), \sup \left(\mu_{q}\right)$ may be interpreted as a measure of persistent excitation (p.e.) associated with this trajectory and with respect to which, given $V(t)$ and $Q(t)$, the lower bound of the observer's exponential convergence rate given by (7) varies monotically. The larger this number, the faster the observer's rate of convergence. This relation can be important for practical purposes when one has the possibility of modifying the state trajectory.

Define the p.s.d. projection matrix operator $\Pi_{y(t)}:=$ $I_{d}-y(t) y^{\top}(t)$ with $y(t) \in \mathbb{R}^{n}$ and such that $|y(t)|=1$ (i.e. $y(t) \in S^{n-1}$ ). This operator plays a central role for the design of the observers proposed in the next section. In particular, when a single source point is used and the body velocity is measured with no bias, we will see that a possible modelling of the system's equations involves the matrices $(A, C(t))=\left(0_{n \times n}, \Pi_{y(t)}\right)$ with $y(t)$ denoting the unit vector along the direction between a source point and the body. Then $\Phi(s, t)=I_{d}$ and the positivity condition upon the Riccati observability Gramian is satisfied provided that $Q(t)$ is chosen larger than an arbitrarily small positive matrix and there exist positive numbers $\delta$ and $\epsilon$ such that

$$
\forall t: \frac{1}{\delta} \int_{t}^{t+\delta} \Pi_{y(s)} d s \geq \epsilon I_{d}>0
$$

In this case it suffices to choose $V(t)$ larger than an arbitrarily small positive matrix to ensure that the solution $P(t)$ to the CRE is bounded and well conditioned. Note that if $u(t)$ is differentiable and $|\dot{u}(t)|$ is bounded then (10) is satisfied provided that $|\dot{y}(t)|$ is regularly larger than some positive number. Note also that the constant matrix $P=I_{d}$ is a solution to the CRE when choosing $V(t)=\Pi_{y(t)} Q(t) \Pi_{y(t)}$ (which is only p.s.d). Although this particular solution holds independently of the persistent excitation condition (10), in view of (5) and (7) the exponential stability of the corre- sponding Riccati observer still depends on the satisfaction of this condition.

The extension of these results to the biased velocity case involves the following technical lemma.

Lemma 2.3: If

1) $A=\left[\begin{array}{cc}0_{n \times n} & I_{d} \\ 0_{n \times n} & 0_{n \times n}\end{array}\right]$
2) $C^{\top}(t) Q(t) C(t)=\left[\begin{array}{cc}\Delta(t) & 0_{n \times n} \\ 0_{n \times n} & 0_{n \times n}\end{array}\right]$ with $\Delta(t)$ a p.s.d bounded matrix-valued function such that, for some $\delta>0$ and $\mu>0: \frac{1}{\delta} \int_{t}^{t+\delta} \Delta(s) d s>\mu I_{d}(\forall t)$

then there exist $\bar{\delta}>0$ and $\epsilon>0$ such that $W_{Q}(t, t+\bar{\delta})>\epsilon I_{d}$ $(\forall t)$.

The proof is given in the appendix B.

\section{OBSERVERS FOR POSITION ESTIMATION FROM DIRECTION MEASUREMENTS}

In the case of a single source point that may arbitrarily be taken as the origin of a fixed frame, the problem consists in estimating the position $x$ of a body (or object) with respect to (w.r.t.) this frame given its velocity $u$ and the measurement of the direction $x /|x|$ between the source point and the body, knowing that the measured velocity may be biased by an unknown constant vector $a$. A situation that may, for instance, explain such a bias is when the body is a vehicle (ship, submarine, aeroplane,...) whose velocity is measured w.r.t. the ambient fluid (water or air), itself moving with a constant unknown velocity (sea-current or wind) w.r.t. an inertial frame. In practice, $x$ will be a twodimensional vector of coordinates (in the $2 \mathrm{D}$, or planar, case) or a three-dimensional vector of coordinates (in the $3 \mathrm{D}$, or spatial, case). For the sake of generality, we assume here that $x \in \mathbb{R}^{n}$, with $n \geq 2$. The corresponding modelling equations are

$$
\begin{aligned}
& \dot{x}=u+a \\
& \dot{a}=0 \\
& 0=\Pi_{y(t)} x
\end{aligned}
$$

with $y(t):=x(t) /|x(t)|$. Let us distinguish two cases, depending on whether the velocity measurement is unbiased, i.e. $a=0$, or is biased by an unknown constant vector $a$.

\section{A. The unbiased velocity case}

In this case the modelling equations can also be written as

$$
\begin{aligned}
& \dot{x}=A x+u \\
& 0=C(t) x
\end{aligned}
$$

with $A=0_{n \times n}$-the $(n \times n)$-dimensional null matrixand $C(t)=\Pi_{y(t)}$. This system can be associated with the following Riccati-like observer

$$
\dot{\hat{x}}=A \hat{x}+u+K(t)(0-C(t) \hat{x})
$$

with the observer gain $K(t)$ calculated as in relation (3) from the solution to the CRE (4). The resulting observer writes as

$$
\dot{\hat{x}}=u-K(t) \Pi_{y(t)} \hat{x}
$$


with $K(t)=k(t) P(t) \Pi_{y(t)} Q(t)$ and $P(t)$ the solution to the CRE

$$
\dot{P}=-P \Pi_{y(t)} Q(t) \Pi_{y(t)} P+V(t)
$$

Since $\dot{\tilde{x}}=(A-K(t) C(t)) \tilde{x}$ the Lyapunov analysis of section II-A applies, and global uniform exponential stability of $\tilde{x}=0$ is obtained provided that $P(t)$ is bounded and well-conditioned. From explanations given in the previous section such is the case if $Q(t)$ and $V(t)$ are chosen positive (and larger than an arbitrarily small positive matrix) or if $V(t)$ is equal to $\Pi_{y(t)} Q(t) \Pi_{y(t)}$ and, most importantly, if the p.e. condition (10) is satisfied. A loose interpretation of this condition is that the body must keep moving and not always in the direction of the source point. Note that, in the case where $V(t)=k_{v} \Pi_{y(t)}$ and $Q(t)=k_{q} I_{d}$, choosing the constant solution $P=\left(k_{v} / k_{q}\right)^{0.5} I_{d}$ simplifies the observer equation to $\dot{\hat{x}}=u-k(t) \sqrt{k_{q} k_{v}} \Pi_{y(t)} \hat{x}$, so that one recovers the solution proposed in [3].

\section{B. The biased velocity case}

In this more difficult case the velocity bias $a$ has to be estimated as well. The modelling equations (11) can be written in the state form as

$$
\begin{aligned}
& \dot{X}=A X+\bar{u} \\
& 0=C(t) X
\end{aligned}
$$

with $X:=\left[x^{\top}, a^{\top}\right]^{\top}$ the $2 n$-dimensional extended state vector, $\bar{u}:=\left[u^{\top}, 0_{1 \times n}\right]^{\top}$, and

$$
A=\left[\begin{array}{cc}
0_{n \times n} & I_{d} \\
0_{n \times n} & 0_{n \times n}
\end{array}\right], C(t)=\left[\begin{array}{ll}
\Pi_{y(t)} & 0_{n \times n}
\end{array}\right]
$$

Consider now the CRE

$$
\dot{P}=A P+P A^{\top}-P C^{\top}(t) Q(t) C(t) P+V(t)
$$

with $P(0)$ a p.d. matrix, $V(t)$ larger than some arbitrarily small positive matrix, and $Q(t)$ a p.d. matrix-valued function larger than an arbitrarily small positive matrix. By application of Lemma 2.3, using the fact that

$$
C^{\top}(t) Q(t) C(t)=\left[\begin{array}{cc}
\Delta(t) & 0_{n \times n} \\
0_{n \times n} & 0_{n \times n}
\end{array}\right]
$$

with $\Delta(t):=\Pi_{y(t)} Q(t) \Pi_{y(t)}$, the solution $P(t)$ to this CRE is bounded and well-conditioned provided that the p.e. condition (10) is satisfied. Denoting the estimate of $X$ as $\hat{X}$ and the estimation error as $\tilde{X}:=X-\hat{X}$, this in turn implies that the following Riccati observer

$$
\dot{\hat{X}}=A \hat{X}+\bar{u}+K(t)(0-C(t) \hat{X})
$$

with $\hat{X}:=\left[\hat{x}^{\top}, \hat{a}^{\top}\right]^{\top}$ and $K(t)=k(t) P(t) C^{\top}(t) Q(t)$ $(k(t) \geq 0.5)$, globally uniformly exponentially stabilizes $\tilde{X}=0$ provided that the p.e. condition (10) is satisfied. This observer can also be written as

$$
\left\{\begin{array}{l}
\dot{\hat{x}}=u+\hat{a}-k(t) P_{11}(t) \Pi_{y(t)} Q(t) \hat{x} \\
\dot{\hat{a}}=-k(t) P_{21}(t) \Pi_{y(t)} Q(t) \hat{x}
\end{array}\right.
$$

with $P_{i j}(i, j \in\{1,2\})$ denoting the block components of $P$ with adequate dimensions.

\section{Extension to multiple direction measurements}

We consider now the problem of estimating a vector $x$ from $l$ direction measurements $y_{i}=\frac{x-z_{i}}{\left|x-z_{i}\right|}, i \in\{1, \ldots, l\}$ with $x$ denoting the vector of coordinates of the position of a body w.r.t. an inertial frame, and $z_{i}$ the known vector of coordinates of the ith source point.

Setting $X:=\left[x^{\top}, a^{\top}\right]^{\top}, \bar{u}:=\left[u^{\top}, 0_{1 \times n}\right]^{\top}$, and $y=$ $\left[\left(\Pi_{y_{1}(t)} z_{1}\right)^{\top}, \ldots,\left(\Pi_{y_{l}(t)} z_{l}\right)^{\top}\right]^{\top}$, one obtains the system

$$
\begin{aligned}
& \dot{X}=A X+\bar{u} \\
& y=C(t) X
\end{aligned}
$$

with

$$
A=\left[\begin{array}{cc}
0_{n \times n} & I_{d} \\
0_{n \times n} & 0_{n \times n}
\end{array}\right]
$$

and

$$
C(t)=\left[\begin{array}{cc}
\Pi_{y_{1}(t)} & 0_{n \times n} \\
\vdots & \vdots \\
\Pi_{y_{l}(t)} & 0_{n \times n}
\end{array}\right]
$$

Consider now the CRE

$$
\dot{P}=A P+P A^{\top}-P C^{\top}(t) Q(t) C(t) P+V(t)
$$

with $P(0)$ a p.d. matrix, $V(t)$ larger than an arbitrarily small positive matrix, and

$$
Q(t)=\left[\begin{array}{cccc}
Q_{11}(t) & 0_{n \times n} & \ldots & 0_{n \times n} \\
0_{n \times n} & Q_{22}(t) & \ldots & 0_{n \times n} \\
\vdots & \vdots & & \vdots \\
0_{n \times n} & 0_{n \times n} & \ldots & Q_{l l}(t)
\end{array}\right]
$$

with $Q_{i i}(t)(i=1, \ldots, l)$ p.d. matrix-valued functions larger than an arbitrarily small positive matrix. The solution $P(t)$ to this equation is bounded and well conditioned provided that the corresponding Riccati observation Grammian $W_{Q}$ is positive. Using the fact that

$$
C^{\top}(t) Q(t) C(t)=\left[\begin{array}{cc}
\Delta(t) & 0_{n \times n} \\
0_{n \times n} & 0_{n \times n}
\end{array}\right]
$$

with $\Delta(t):=\sum_{i=1}^{l} \Pi_{y_{i}(t)} Q_{i i}(t) \Pi_{y_{i}(t)}$, this condition is again satisfied by application of Lemma 2.3 provided that, for some $\delta>0$ and for all $t>0, \frac{1}{\delta} \int_{t}^{t+\delta} \bar{\Delta}(s) d s$, with $\bar{\Delta}(t):=\sum_{i=1}^{l} \Pi_{y_{i}(t)}$, is greater than an arbitrarily small p.d. matrix. This p.e. condition clearly points out the interest of using multiple direction measurements in order to weaken, or even remove, conditions upon $x$ and its time-variations. For instance, in the $3 \mathrm{D}$ case $(n=3)$, if $l \geq 2$ this p.e. condition is satisfied provided that the body is periodically not aligned with all the source points. If three or more source points are not aligned, then this condition is automatically satisfied independently of $x$ and its time-variations.

A Riccati observer associated with this system is

$$
\dot{\hat{X}}=A \hat{X}+\bar{u}+K(t)(y-C(t) \hat{X})
$$

with $\hat{X}:=\left[\hat{x}^{\top}, \hat{a}^{\top}\right]^{\top}$ and $K(t)=k(t) P(t) C^{\top}(t) Q(t)$ $(k(t) \geq 0.5)$. One easily verifies that this observer can also 
be written as

$$
\left\{\begin{array}{l}
\dot{\hat{x}}=u+\hat{a}-k(t) P_{11}(t)\left(\sum_{i=1}^{l} \Pi_{y_{i}(t)} Q_{i i}(t)\left(\hat{x}-z_{i}\right)\right) \\
\dot{\hat{a}}=-k(t) P_{21}(t)\left(\sum_{i=1}^{l} \Pi_{y_{i}(t)} Q_{i i}(t)\left(\hat{x}-z_{i}\right)\right)
\end{array}\right.
$$

From what precedes this observer globally exponentially stabilizes $\tilde{X}=0$ if the previously evoked p.e. condition is satisfied.

\section{Remarks:}

- In the 3D-case, if $l \geq 2$ and the matrix $\bar{\Delta}(t)$ is positive, and if the body moves with a constant unknown velocity, the above observer provides also an estimation of this velocity. To this aim it suffices to set $u=0$ in the algorithm. The term $\hat{a}$ is then an estimate of the body velocity that is equal to $a$ in this case.

- In the unbiased case where $a=0$ and the body velocity $u$ is measured, the calculation of $\hat{a}$ is superfluous and the above observer reduces to

$$
\dot{\hat{x}}=u-k(t) P(t)\left(\sum_{i=1}^{l} \Pi_{y_{i}(t)} Q_{i i}(t)\left(\hat{x}-z_{i}\right)\right)
$$

with $P(t)$ the solution to the CRE

$$
\dot{P}=-P \Delta(t) P+V(t)
$$

A particular solution to this latter equation, obtained by choosing $V(t)=P(0) \Delta(t) P(0)$, is any constant p.d. matrix.

\section{Simulations}

We estimate the position of a possibly moving body from one or two sources. Two scenarios are considered with a minimal number of source points ensuring uniform observability. In the first scenario the body oscillates along a line segment with its position given by $x(t)=(20 \cos t-15,0,4)^{\top}$. In this case a single source point, here chosen at the origin of the inertial frame, suffices to grant uniform observability. In the second scenario the body is motionless and located at $x=(5,0,4)^{\top}$. In this case a second source point, here chosen with coordinates $(3,3,0)^{\top}$ in the inertial frame, is required to grant uniform observability. For both scenarios the body velocity measurement is corrupted by the constant bias $a=(0.33,0.66,0.99)^{\top}$. Initial state estimates are $\hat{x}(0)=(4,6,12)^{\top}$ and $\hat{a}(0)=(0,0,0)^{\top}$. The scalar gain $k(t)$ involved in the CRE is set to one, as for a Kalman filter, and the solution to the CRE is initialized with $P(0)=100 I_{6}$. Simulations are first carried out with noise-free measurements, to validate theoretical exponential stability results, then with measurements corrupted by noise, to illustrate the resulting (and inevitable) slight degradation of the observers following the transient phase when the estimation errors become small but no longer converge to zero. Concerning the body velocity measurement, additive and independent zero mean Gaussian noise components with standard deviations equal to $0.1 \mathrm{~m} / \mathrm{s}$ are used. As for the direction measurements, they are calculated from the body position corrupted by additive and independent zero mean Gaussian noise components with standard deviations equal to $0.05 \mathrm{~m} / \mathrm{s}$. The matrix $V$ (i.e. the state noise variance in the Kalman filtering terminology) involved in the CRE is chosen equal to $0.01 \operatorname{diag}\{1,1,1,0,0,0\}+\epsilon_{v} I_{6}$, with $\epsilon_{v}=0.001$ to ensure that the matrix is positive definite. As for the matrix $Q$, i.e. the inverse of the output noise variance in the Kalman filtering terminology, we have used $Q_{i i}=1.5 I_{3}, \forall i=1 \ldots l$ with $l$ denoting the number of source points.

Figures 1(a)-1(c) and Figures 2(a)-2(c) illustrate the performance of the observer in the ideal noise-free case for scenarios 1 and 2 respectively. Sub-figures $(a)$ show the time-evolution of the logarithm of the Lyapunov function associated with the observer. The mean slope of the curve is proportional to the rate of exponential convergence of this function to zero. Sub-figures $(b)$ and $(c)$ show the time-evolution of the estimation errors and their exponential convergence to zero. The last figures 1(d)-1(e) and 2(d)-2(e) show the time-evolution of these errors in the case of noisy measurements.

\section{CONCLUDING REMARKS}

Riccati observers for the estimation of a body position from direction measurements and the knowledge of the body velocity have been derived. Even when the body velocity is biased by an unknown constant vector, these observers ensure global exponential stability of zero estimation errors under uniform observability conditions that have been worked out in relation to the number of source points and the body motion. A logical prolongation of this work is the derivation of Riccati observers for the estimation of the complete body pose (position and orientation). However, due to the specific structure of the group of rotations, exact linearisation of the problem is then no longer possible and globally convex cost functions do not exist. As a consequence Riccati observers for pose estimation, and corresponding Extended Kalman Filters (EKF), have to be derived from linear approximations of the system state and output equations. This also implies that only local exponential stability of zero estimation errors can be achieved. An important complementary issue, also in the prolongation of the present work, is the characterisation of uniform observability conditions under which this latter property is granted. We foresee several other possible extensions. Let us just mention position estimation from range measurements, in relation to the recent works of Batista et al. [7], [8] for the non-static case, visionbased robotic applications involving the control of the body position from estimates provided by Riccati observers, and a deterministic approach to Simultaneous Localication and Mapping (SLAM) that could usefully complement existing studies on the subject.

\section{ACKNOWLEDGMENTS}

This work was supported by the ANR-ASTRID project SCAR "Sensory Control of Unmanned Aerial Vehicles", the ANR-Equipex project "Robotex". 


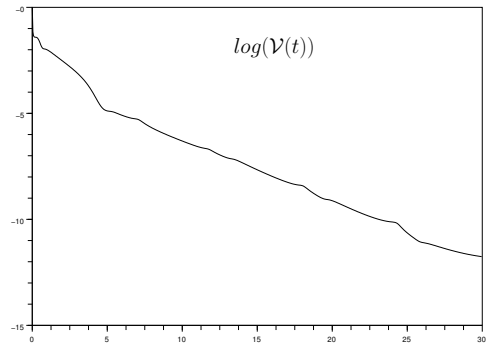

(a) Time evolution of the Lyapunov function logarithm

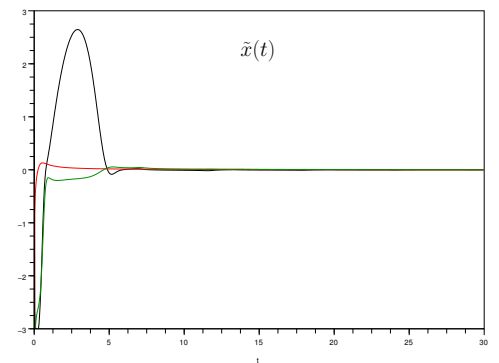

(b) Time evolution of position estimation errors

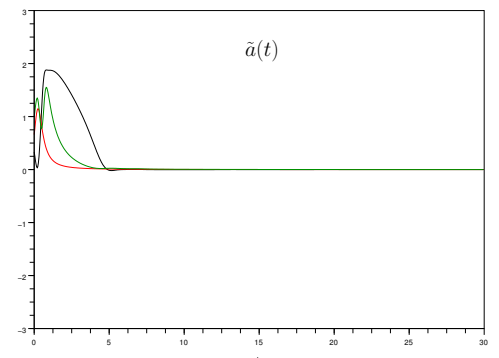

(c) Time evolution of velocity bias estimates

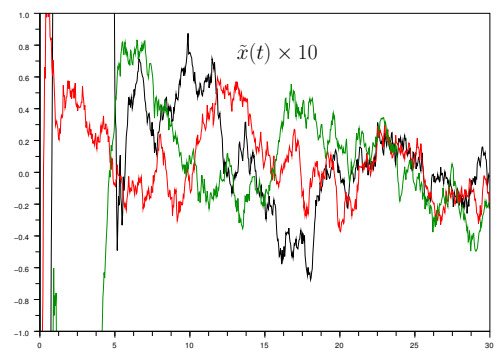

(d) Time evolution of position estimation errors in the case of noisy measurements

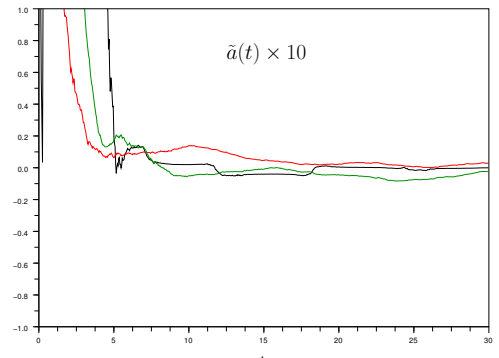

(e) Time evolution of position estimation errors in the case of noisy measurements

Fig. 1. Scenario 1

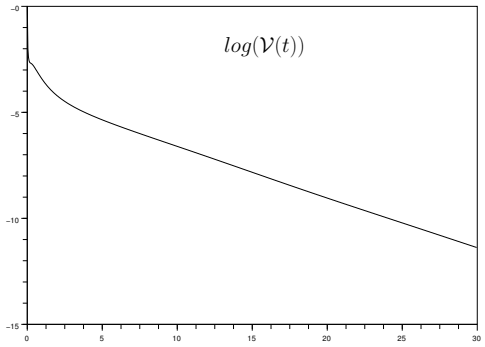

(a) Time evolution of the Lyapunov function logarithm

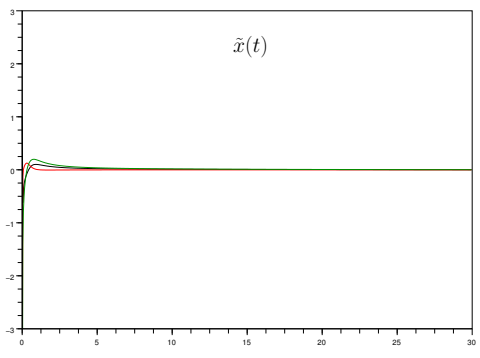

(b) Time evolution of position estimation errors

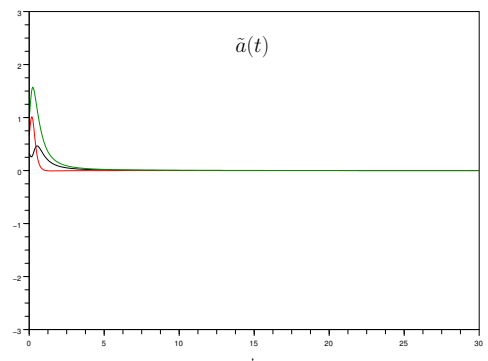

(c) Time evolution of velocity bias estimates

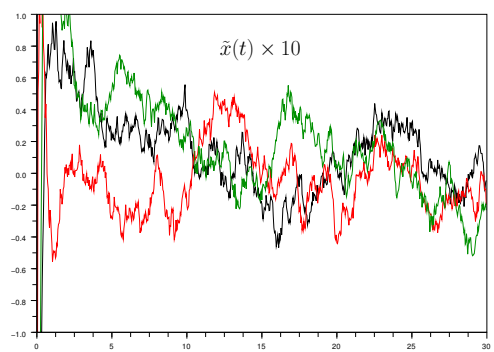

(d) Time evolution of position estimation errors in the case of noisy measurements

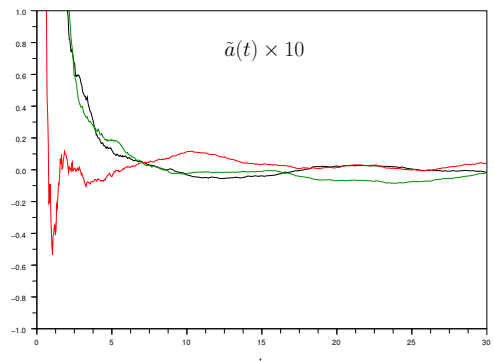

(e) Time evolution of position estimation errors in the case of noisy measurements

Fig. 2. Scenario 2 


\section{APPENDIX}

\section{A. Proof of lemma 2.1}

Recall that, as long as $P(t)$ is defined and p.d., its trace is the sum of its eigenvalues. Accordingly, since the eigenvalues of $P^{-1}(t)$ are the inverse of the ones of $P(t)$, the trace of $P^{-1}(t)$ is the sum of the inverse of the eigenvalues of $P(t)$. To prove that $P(t)$ is well-defined for $t \in[0,+\infty)$ and is p.d. it suffices to show that neither the trace of $P(t)$ nor the trace of $P^{-1}(t)$, which are initially positive (since $P(0)$ is p.d. by assumption), can tend to infinity in finite time. Indeed, this implies that none of the eigenvalues of $P(t)$ can either reach zero or tend to infinity in finite time. To this aim, it suffices to show that neither $\operatorname{tr}\left(P(t)\right.$ nor $\operatorname{tr}\left(P^{-1}(t)\right)$ can grow faster than exponentially, so that divergence in finite time is not possible.

Let us set $x=\operatorname{tr}(P)$. In view of (4), and since $\operatorname{tr}\left(P(t) C^{\top}(t) Q(t) C(t) P(t)\right) \geq 0$, one has

$$
\dot{x} \leq \operatorname{tr}(A P)+\operatorname{tr}\left(P A^{\top}\right)+\operatorname{tr}(V)
$$

Let $|A(t)|$ denote the spectral norm of $A(t)$. By assumption it is bounded by some positive number $k_{a}$. Similarly, $\operatorname{tr}(V(t))$ is bounded by a positive number $v$. Since $P$ is p.s.d., $|\operatorname{tr}(A P)|=\left|\operatorname{tr}\left(P A^{\top}\right)\right| \leq|A| \operatorname{tr}(P)$ and the previous inequality yields

$$
\dot{x} \leq 2 k_{a} x+v
$$

This inequality in turn implies that $x(t) \leq(x(0)+$ $\left.\frac{v}{2 k_{a}}\right) \exp \left(2 k_{a} t\right)-\frac{v}{2 k_{a}}, \forall t \geq 0$.

Similar arguments applied to $y=\operatorname{tr}\left(P^{-1}\right)$ yield

$$
\begin{aligned}
\dot{y} & \leq\left|\operatorname{tr}\left(P^{-1} A\right)\right|+\mid \operatorname{tr}\left(A^{\top} P^{-1}\right)+\operatorname{tr}\left(C^{\top} Q C\right) \\
& \leq 2 k_{a} y+\bar{\mu}_{q}
\end{aligned}
$$

with $\bar{\mu}_{q}$ denoting the supremum of $\operatorname{tr}\left(C^{\top}(t) Q(t) C(t)\right)$. Therefore, $y(t) \leq\left(y(0)+\frac{\bar{\mu}_{q}}{2 k_{a}}\right) \exp \left(2 k_{a} t\right)-\frac{\bar{\mu}_{q}}{2 k_{a}}, \forall t \geq 0$.

\section{B. Proof of lemma 2.3}

From the expression of the matrix $A$ one has

$$
\Phi(s, t)=\exp (A(s-t))=\left[\begin{array}{cc}
I_{n \times n} & (s-t) I_{n \times n} \\
0_{n \times n} & I_{n \times n}
\end{array}\right]
$$

so that

$$
\begin{aligned}
W_{Q}(t, t+\bar{\delta}) & :=\frac{1}{\delta} \int_{t}^{t+\bar{\delta}} \Phi^{\top}(s, t) C^{\top}(s) Q(s) C(s) \Phi(s, t) d s \\
& =\frac{1}{\delta} \int_{t}^{t+\bar{\delta}}\left[\begin{array}{cc}
\Delta(s) & (s-t) \Delta(s) \\
(s-t) \Delta(s) & (s-t)^{2} \Delta(s)
\end{array}\right] d s
\end{aligned}
$$

Let us make a proof by contradiction and assume that $\forall \epsilon>0$, $\forall \bar{\delta}>0, \exists x \in S^{2 n-1}$ such that $x^{\top} W_{Q}(t, t+\bar{\delta}) x<\epsilon$. Then, in view of the above expression of $W_{Q}(t, t+\bar{\delta})$

$$
\begin{aligned}
& x^{\top} W_{Q}(t, t+\bar{\delta}) x= \\
& \frac{1}{\delta} \int_{t}^{t+\bar{\delta}}\left(x_{1}+(s-t) x_{2}\right)^{\top} \Delta(s)\left(x_{1}+(s-t) x_{2}\right) d s<\epsilon
\end{aligned}
$$

with $x=\left[x_{1}^{\top}, x_{2}^{\top}\right]^{\top}$. It is clear that $x_{2} \neq 0$ since otherwise, the above integral is equal to $\frac{1}{\delta} \int_{t}^{t+\bar{\delta}} x_{1}^{\top} \Delta(s) x_{1} d s>\mu$ with $\bar{\delta}=\delta$. Moreover, since $\Delta(s)$ is a p.s.d. matrix, $x^{\top} W_{Q}(t, t+$ $\bar{\delta}) x$ is larger than

$$
\begin{aligned}
& \gamma(t, \delta, \bar{\delta}):=\frac{1}{\delta} \int_{t+\bar{\delta}-\delta}^{t+\bar{\delta}}\left(x_{1}+(s-t) x_{2}\right)^{\top} \Delta(s)\left(x_{1}+(s-t) x_{2}\right) d s \\
& \quad=\frac{1}{\delta} \int_{\bar{\delta}-\delta}^{\bar{\delta}} s^{2}\left(\frac{x_{1}}{s}+x_{2}\right)^{\top} \Delta(t+s)\left(\frac{x_{1}}{s}+x_{2}\right) d s
\end{aligned}
$$

when $\bar{\delta}>\delta$. From the latter equality we also have

$\gamma(t, \delta, \bar{\delta}) \geq \frac{(\bar{\delta}-\delta)^{2}}{\bar{\delta}} \int_{\bar{\delta}-\delta}^{\bar{\delta}}\left(\frac{x_{1}}{s}+x_{2}\right)^{\top} \Delta(t+s)\left(\frac{x_{1}}{s}+x_{2}\right) d s$

which, using the assumptions upon $\Delta(t)$, in turn implies that $\gamma(t, \delta, \bar{\delta})$ grows to infinity when $\bar{\delta}$ tends to infinity. In particular there exists $\bar{\delta}_{0}>0$ such that $\gamma(t, \delta, \bar{\delta})>\epsilon$ when $\bar{\delta}>\bar{\delta}_{0}$. This contradicts our initial assumption since $x^{\top} W_{Q}(t, t+\bar{\delta}) x \geq \gamma(t, \delta, \bar{\delta})$.

\section{REFERENCES}

[1] R. Haralick, C. Lee, K. Ottenberg, and M. Nlle, "Review and analysis of solutions of the three point perspective pose estimation problem," International Journal of Computer Vision, vol. 13, no. 3, pp. 331-356, 1994.

[2] P. Batista, C. Silvestre, and P. Oliveira, "Globally exponentially stable filters for source localization and navigation aided by direction measurements," Systems \& Control Letters, vol. 62, no. 11, pp. 1065-1072, 2013.

[3] F. L. Bras, T. Hamel, R. Mahony, and C. Samson, "Observer design for position and velocity bias estimation from a single direction output," in 54th IEEE International Conference on Decision and Control (CDC), 2015.

[4] R. Kalman, "A new approach to linear filtering and prediction problems," Transactions of the ASME-Journal of Basic Engineering, vol. 82, pp. 35-45, 1960.

[5] A. Jazwinski, Stochastic process and filtering theory. Mathematics in Science and Engineering, Academic Press Inc., 1970, vol. 64.

[6] M. Pengov, E. Richard, and J.-C. Vivalda, "On the boundedness of the solutions of the continuous riccati equation," J. of Inequal. and Appli., vol. 6, pp. 641-649, 2001.

[7] P. Batista, C. Silvestre, and P. Oliveira, "Sensor-based long baseline navigation: observability analysis and filter design," Asian J. Control, vol. 16, pp. 974-994, 2014.

[8] __ "Tightly coupled long baseline/ultra-short baseline integrated navigation system," International Journal of Systems Science, no. aheadof-print, pp. 1-19, 2014. 